\title{
Psychiatric emergencies in people with a mental handicap
}

\author{
DORA KoHEN, Senior Lecturer and Honorary Consultant, Academic Department of \\ Psychiatry, Charing Cross Westminster Medical School, Fulham Palace Road, \\ London W6 8RP
}

With the implementation of community care and resettlement programmes, the extent, frequency and the management of emergencies in the mentally handicapped population have attracted a good deal of attention. Among the issues of importance are crisis in the community and unnecessary and repeated hospital admission; inappropriate emergency referrals leading to abuse of services; the need for emergency services and the importance of short term admission facilities (Nolan et al, 1992). While the number of psychiatric emergencies in people with mental handicap is not large, the trend towards living in the community has brought the need to monitor such referrals.

The aim of this study is to describe emergency referrals to a consultant psychiatrist in mental handicap in central London and to clarify the psychiatric emergency needs of the mentally handicapped population living in this community.

\section{The study}

All referrals to the consultant psychiatrist in mental handicap, the only doctor taking such referrals, for 12 months from 1 April 1991 to 31 March 1992, in the Royal Borough of Kensington and Chelsea (a population of 127,000 of whom 362 are registered as having a mental handicap), were studied prospectively. Not all are known to the consultant psychiatrist and it is usually the community mental handicap team (CMHT) and the local social services that provide the day to day care.

The consultant psychiatrist receives referrals from the community team, general practitioners, social services, the families, and other psychiatrists. Most are requests for out-patient appointments or domiciliary visits for patients with psychiatric disorders, such as behavioural problems, follow-up of epilepsy, minor psychiatric morbidity, eating disorders, and sexual problems.

Cases were classified as emergencies when the consultant psychiatrist was bleeped during working hours with a request for an emergency assessment within 24 hours. Medical emergencies requiring urgent input from non-psychiatric generic services have not been included.
Data were collected prospectively using a 40-item questionnaire concerning type of the emergency, social conditions, housing, family involvement, existence of a support system including input from social services; daytime activity; presenting psychiatric problem and medical complaint, diagnosis, action taken and management.

\section{Findings}

Ninety-seven referrals occurred during the study period. Thirty-five were from community nurses; 15 from the family; 15 from the GP; 11 from another consultant psychiatrist; 9 from key workers at hostels or homes; one from the day centre and one was a self referral.

Sixteen of the cases were classified as emergency referrals. All were referred by the community nurses who, after immediate evaluation, contacted the psychiatrist. All except one were living in the parental or own home with extensive family support and involvement; one lived in a voluntary sector hostel. Ages ranged from 19 to 84 (mean 51). Ten (six men and four women) had a mild to moderate level of handicap and six (three men and three women) severe to profound. Each had been in close contact with the service and were well known to the community services. All had at least two sessions per week of daytime activities and all were supported by social services in adult training services and programmed day activities; four had regular respite care. The emergency referrals were preceded by a daytime crisis where the carers could not cope and needed professional input. The presenting complaints were uncontrollable epileptic seizures (in five) and aggressive or self-injurious behaviour, or both, (in 11). In cases with epilepsy, the belief that the patient needed further medical attention was prominent.

Among cases with aggressive behaviour, three patients required surgical intervention for minor suturing. The rest could not be contained in the house or hostel where they lived without alteration in their medication, change in the management of their behavioural problems and outside specialist input.

Of the epileptic patients with exacerbated seizures, only one had had a series of non-epileptic fits 
following personal and familial distress. Of the other four, one had had a change in lifestyle and forgotten to take his anti-epileptics, and had had three tonicclonic seizures in 24 hours; one had taken twice the prescribed dose accidentally and was drowsy and confused; one person went into status epilepticus although blood biochemistry showed his anti-epileptics to be within therapeutic limits; and one person showed fears, delusions and a reversible but alarming hemiparesis following two seizures within $\mathbf{4 8}$ hours.

Of the 11 cases with behavioural aggressive problems, five had depression or anxiety and depression, four behavioural problems, one schizophrenia and one had tuberous sclerosis, the emergency situation being caused by aggression, triggered by increased intracranial pressure due to multiple brain tumours which required a ventricular shunt operation.

Four cases had behavioural disorder without further specific diagnosis. Three had a severe/ profound mental handicap which caused difficulties in assessment and one man had previous selfinjurious behaviour which fluctuated situationally. The four cases with behavioural problems were referred to the clinical psychologist, speech and occupational therapist and have specific programmes to modify their behaviour. Two had a reduction in psychotropic medication and two an increase. Three were offered places at the respite care unit and have regular community nurse visits.

Nine cases had a previous DSM-III-R diagnosis and were seen by the psychiatrist when the emergency occurred. The remaining seven cases were not known to the psychiatrist but had been in contact with the day services and community team.

Three cases required hospital admission while 13 of the emergencies were managed in the community with the input of psychiatrists, community mental handicap team, community nurses and the respite care services.

Following emergency referrals there was generally an increase in dose and number of psychotropic medication. The five people with depression were started on antidepressants and five patients had a decrease or no change in their psychotropics.

The patients who went through the emergency process are still followed up as out-patients. Two of the hospital admissions have been discharged but the patient with tuberous sclerosis is still in an admission unit of a large mental handicap hospital. All patients are seen by members of the community team.

None of the emergency cases re-presented as emergencies during the follow-up period.

\section{Comment}

Only $17 \%$ of referrals of the mentally handicapped population in Kensington and Chelsea proved to be psychiatric emergencies. Each was known to health and social services, but behavioural disorders and epilepsy continued to be a source of emergency referrals and crisis.

The small number of borough residents with mental handicap and lack of large number of recent discharges from mental handicap hospitals may have affected the levels of emergencies and the type of problem. There are 110 people from this borough living in eight different large mental handicap hospitals around London. The medical and psychiatric problems of this population will obviously change the pattern of emergency referrals when they are resettled in this borough.

The majority of emergency referrals presented as behavioural problems, physical aggression and selfinjurious behaviour. This picture reflects the true nature of complaints in the population with a mental handicap. Where verbal expression is limited, and affective and cognitive changes not readily recognisable, patients resort to behavioural expressions. In houses and respite care units, the high turnover of staff who lack basic knowledge about the client group and dismiss complaints as idiosyncratic, and the lack of personal attention, together with anxiety and boredom, may be a contributory factor.

Behavioural disorder, aggression and self-injury may be a common presentation in schizophrenia, depression, bipolar affective disorder and minor psychiatric morbidity. Depression in people with severe to profound mental handicap can be difficult to diagnose and present with restlessness, irritability and mobility changes.

Epilepsy is usually well controlled but this survey shows that it may always need immediate medical attention and will remain a potential source for emergencies.

Even after extensive evaluation one quarter of the emergency cases could be diagnosed only as behavioural disorder. Difficulties in assessment and lack of reliable diagnostic instruments for this group contribute to the difficulties.

Behaviour problems and their expression should be highlighted and care staff in houses or hostels and respite care units trained in their recognition and management. Access to a behavioural unit with trained professionals, including psychologist and community nurses, should become a priority in each district.

The increase in psychotropic medication and changes in antiepileptics highlight the importance of monitoring neuroleptic and antiepileptic drugs to help prevent emergencies.

Regular visits by trained professionals without waiting for formal referrals and training programmes for parents, staff and voluntary organisations may speed up referrals before the cases become emergencies. 
The survey also shows that the community team, especially the community nurses, have good understanding of emergency, where all their referrals showed valid needs for requests. Possible medical or psychiatric emergencies should not deter professionals from resettlement programmes or community care. Most can be dealt with in community settings. A trained, experienced and harmonious community team, a well organised back-up service of generic

hospital beds and admission-assessment units for mentally handicapped people will deal with emergencies effectively.

\title{
Reference
}

Nolan, M. E., Radakrishnan, G. \& Lewis, J. (1992) Generic services for people with a mental handicap. Psychiatric Bulletin, 16, 212-213.

\section{A Nigerian psychiatric emergency service: factors associated with utilisation}

\author{
R. A. Adeniran, Registrar, North Wales Hospital, Denbigh, Clwyd LL16 5SS; and \\ O. A. Suuwola, Professor of Psychiatry, Neuropsychiatric Hospital \& WHO \\ Collaborating Centre for Research \& Training in Mental Health, Abeokuta, \\ Nigeria
}

The psychiatric emergency service is a major focal point of entry into the mental health system. Depending on the health care policy and other sociocultural factors, the pattern of attendance in a psychiatric emergency unit tends to differ from one country to another (Bassuk et al, 1983).

The psychiatric emergency service often reflects the ideology and provision of mental health services in a community, while the nature of the population utilising it reflects the psychiatric trends in that community. Various studies about the pattern of utilisation of psychiatric emergency services in many countries have shown that for a large number of patients, the psychiatric emergency unit is the source of primary care. There has been no such study in Nigeria and mental health is now part of the primary health care in the country. In planning and providing mental health care at the grass roots, there is a need to know the nature and extent of psychopathology in the population and the pattern of utilisation of existing services.

This study was conducted to identify the population that make use of a Nigerian psychiatric emergency unit and the factors associated with this. Since there is now a focus on mental health at the primary care level, it is hoped that our findings would supplement existing information to assist planning and service provision.

\section{The study}

The study was conducted in the assessment unit of the Neuropsychiatric Hospital, Aro, Abeokuta, Nigeria. It is Nigeria's foremost psychiatric hospital with 526 beds and no defined catchment area. The assessment unit has an open door policy. It serves as the first contact point for all patients coming to the hospital for the first time and provides a 24-hour service, including emergency services seven days of the week.

All consecutive patients seen at the assessment unit over a two-month period between April and June 1989 were included in the study. Data were collected on case histories, sociodemographic status, psychiatric and physical assessment. Psychiatric diagnoses were made according to the first two axes of the Diagnostic and Statistical Manual, 3rd Edition. For the purpose of this study, psychiatric emergencies were operationally defined as follows:

(a) all patients who presented after normal working hours

(b) all patients who presented on weekends and public holidays

(c) all patients who presented during normal working hours and had to be referred on the same day to the out-patient clinic. 